\title{
LA SOCIABILIDAD EN LA HISTORIA POLÍTICA DEL OCHOCIENTOS: UN RECORRIDO POR LA HISTORIOGRAFÍA ITALIANA ${ }^{1}$
}

Rafael Zurita Aldeguer

Tras la estela de las obras de Agulhon, la historiografía italiana sobre la sociabilidad ha experimentado un importante desarrollo desde finales de la década de 1980. Centrada en la Historia Contemporánea, y de forma especial en el siglo XIX, la producción historiográfica del país trasalpino destaca por prestar una especial atención a los aspectos políticos de la sociabilidad. Por un lado, analiza la sociabilidad de las elites, entendida como lugar de agregación prepolítica y de ejercicio de prerrogativas propio de la ciudadanía, al menos hasta la ley electoral de 1882. Junto a ello, estudia las formas organizativas de las clases populares, evidenciando que aquéllas actúan como vehículo de transmisión y socialización de contenidos políticos y estableciendo un nexo entre democracia y asociacionismo. Así, la distinción entre sociabilidad "integradora» $\mathrm{y}$ "opositora» puede resultar clave para interpretar el alcance de los fenómenos asociativos en el contexto de los procesos de nacionalización y politización de la sociedad italiana del Ochocientos. Resulta patente que la dimensión nacional, asumida por las instituciones y por la política en el Estado liberal, aparece como el escenario donde los estudios sobre asociacionismo y sociabilidad son claves para la renovación de la historia política².

En 1988 se publican las primeras monografías que dialogan con el modelo "agulhoniano", como la de Soldani sobre la pequeña comunidad urbana de Prato, en Toscana, en la transición entre el Antiguo Régimen y los primeros años del siglo XX. En ella se relacionan distintas instituciones y factores eco-

1. El autor quiere expresar su agradecimiento al profesor Maurizio Ridolfi por las orientaciones y sugerencias que ha vertido sobre este texto. Una primera versión del mismo se presentó en el Seminario El estudio de la sociabilidad en España, Francia e Italia: lineas de investigación y nuevas perspectivas, que tuvo lugar en la Universidad de Valladolid en noviembre de 2003.

2. Cerast, L., "Identità sociali e spazi delle associazioni. Gli studi sull'Italia liberale», Memoria $e$ Ricerca, n. 10 (1997), pp. 123-146. 
nómicos, observando la influencia que ejercen sobre la mutación de las formas de sociabilidad y sobre la politización de las clases populares ${ }^{3}$. En esta línea de investigación, los efectos de 1848 son objeto de investigación preferente en el libro de Rizzi, quien analiza el impacto de la revolución y de la República Romana en la politización de los círculos populares en las comunidades rurales del Lacio ${ }^{4}$. Un tercer espacio que centra la atención de los historiadores en estos años es la Romaña, destacando de forma especial Il circolo virtuoso, de Ridolfif ${ }^{5}$. El autor se detiene en la difusión de la política en la segunda mitad del siglo XIX, hasta la entrada en escena de la moderna "forma-partido"; por eso extiende su investigación hasta las asociaciones no directamente políticas que le permiten descender a la historia social de la cultura de la «vida cotidiana» y redescubrir, a ese nivel, la relación con la política y con la "subcultura» política. Dimensiones socio-culturales y político-institucionales vienen a entrelazarse con la afirmación de prácticas asociativas no muy elitistas y con la aparición de las primeras formas de participación popular en la vida política de la Italia liberal, que resulta así mejor definida en su dimensión de sociedad de transición. Poniendo en el centro de su análisis el universo democrático y socialista, Ridolfi verifica los mecanismos que modifican las «reglas del juego» tras 1848 y, en particular, tras la Unificación, en coincidencia con los cambios en las normas electorales y con la apertura de nuevos espacios de participación y de representación, tanto en el ámbito nacional como local. Es entonces cuando las redes del asociacionismo democrático de carácter social, cultural y prepolítico asumen una función de «integración», convirtiéndose en un eficaz vehículo de movilización política y electoral. Dicho proceso tiene lugar en áreas como la Padania, donde las redes de las estructuras de la sociabilidad eran más fuertes y extensas. Y, a la postre, surge la pregunta sobre los efectos que produjo en la mentalidad de las clases populares la dicotomía entre instituciones públicas con sufragio limitado y ordenamiento asociativo voluntario de carácter democrático.

Con el inicio de la década de 1990 aparecen en la historiografía italiana los primeros planteamientos teóricos ${ }^{6}$, que ponen de manifiesto que la aplicación del concepto de sociabilité en Italia, como impulsor de una nueva historia política, tiene un problema de fondo distinto del de Francia, como es el del

3. Soldanl, S., "Vita quotidiana e vita di società in un centro industrioso", en Morr, G. (a cura di), Prato. Storia di una città, 3, Il tempo dell'industria (1815-1943), Comune di Prato-Le Monnier, 1988, pp. 663-806.

4. Rrzzi, F, La coccarda e le campane. Comunità rurali e Repubblica Romana nel Lazio (1848-1849), Milano, Franco Angeli, 1988.

5. Ridolfi, M., Il circolo virtuoso. Sociabilità democratica, associazionismo e rappresentanza politica nell' $O t$ tocento, Firenze, Centro editoriale toscano, 1990. Un primer avance sobre esta región en "Associazionismo e forme di sociabilità in Emilia-Romagna tra ' 800 e ' 900 ", monográfico de Il Bollettino del Museo del Risorgimento di Bologna, n. XXXII-XXXIII (1987-1988).

6. "Sociabilità e associazionismo in Italia: anatomia di una categoria debole", intervenciones de Maria Malatesta, Alberto Banti, Marco Meriggi, Gilles Pécout y Simonetta Soldani, en Passato e Presente, n. ${ }^{\circ} 26$ (1991), pp. 17-42. «Sociabilité/Sociabilità nella storiografia dell'Italia dell'Ottocento", monográfico de Dimensioni e problemi della ricerca storica, n. 1 (1992). 
tiempo, el espacio y los actores, tanto del proceso de politización como de nacionalización. Malatesta piensa que para hablar de sociabilidad en Italia es necesario dejar clara la transformación geográfica del término en un país distinto de donde ha nacido, y para ello resulta imprescindible distinguir sus dos componentes fundamentales: por un lado, es una teoría de las formas sociales, en donde el cambio social viene por la imitación de las formas asociativas y de la política; por otra parte, es una metodología, es decir, se trata de una historia regional donde se analiza la estructura social y sus cambios en el seno de las asociaciones. Y este segundo elemento es, según Malatesta, el que ha primado en Italia ${ }^{7}$.

En el concepto de sociabilidad está presente la relación entre formas colectivas de socialización y afirmación del aprendizaje político. Binomio que necesita una nueva aproximación a la historia política para un período caracterizado por la diferenciación entre sociedad civil, Estado y sistema político, de manera que la cuestión que se plantea es el nexo que puede establecerse entre sociabilidad, sociedad civil y proceso de politización en Italia antes y después de la unidad ${ }^{3}$. A este respecto, Pécout afirma que la primera especificidad de la Italia postunitaria es el rol jugado por el Estado liberal a la hora de favorecer la correspondencia entre valores nuevos de la política y sociabilidad. Un ejemplo de ello lo encontramos en un tipo de sociabilidad "institucional» nacida durante el Risorgimento, las sociedades de Tiro, ligadas al voluntariado radical de Garibaldi, y que en 1861 se convierten en una institución nacional. Se trata de asociaciones deportivas y militares que son, al mismo tiempo, las primeras células ideales de la "Nación armada", estableciendo una relación ambigua entre lugares de asociación y lugares de participación ${ }^{9}$. Junto a esta cuestión, conviene precisar además que, mediante el estudio de la sociabilidad, se puede llegar al análisis de la formación y de la circulación del discurso político, superando la distancia entre sociedad e instituciones y reencontrando en lo social y en las relaciones de grupo los elementos y los sujetos de la política ${ }^{10}$.

Otro tema que también ha preocupado a los historiadores italianos es el vínculo entre la sociabilidad y la estratificación de la sociedad. Es decir, si sus formas de expresión, en el paso de un grupo social a otro, se basan en la mimesis, y sólo después se distinguen en los contenidos y en las funciones, ¿cómo puede representar la categoría de la sociabilidad la dialéctica «alto/bajo» de la modalidad asociativa en la Italia del siglo XIX? Para Soldani, la característica principal de la sociabilidad en el siglo XIX son los nexos verticales, que quedan patentes en las sociedades de socorro mutuo de Toscana, en las que se desarrolla una solidaridad nutrida por fuertes influjos paternalistas y nobiliarios, y perfecta-

7. «Sociabilità e associazionismo...», p. 19.

8. Idem, p. 26.

9. Idem, p. 28. Véase, PeCout, G., "Les sociétés de tir dans l'Italie unifié de la seconde moitié du XIX siècle", Mélanges de l'Ecole Française de Rome, n. ${ }^{\circ} 2$ (1990), pp. 533-676.

10. Ciuffolett1, Z., «Le forme della sociabilità e i processi di politicizzazione», en Dimensioni e problemi della ricerca storica, $\mathrm{n} .{ }^{\circ} 1$ (1992), pp, 49-54. 
mente funcional en una economía y en una sociedad en la que todos hablan el mismo lenguaje, desde las relaciones de producción hasta las creencias religiosas. Meriggi, en cambio, discrepa, al señalar que la dialéctica "alto/bajo" está más caracterizada por el signo de la separación que el de la integración, y que, en la Italia del Ochocientos, asociarse es un privilegio reservado a unos pocos ${ }^{11}$. Por su parte, Malatesta opina que determinados contextos económico-sociales pueden producir formas específicas de sociabilidad no siempre reducibles al esquema de los «vasos comunicantes». Si este diseño funciona sobre el plano de la transmisión de las formas asociativas, puede sufrir variaciones sobre el de los contenidos. Éste es uno de los atributos fundamentales de la sociabilidad: el cambio en los contenidos mientras permanecen las formas. Así, pese al origen burgués de las sociedades de socorro mutuo, en muchas de ellas tiene lugar una transformación de la lógica asistencial en resistencia obrera ${ }^{12}$. Para Banti, el campo de la sociabilidad experimenta un cambio en torno a la década de 1880 . $\mathrm{Si}$, hasta ese momento, unas asociaciones tienden a definir límites «horizontales» de la estratificación, otras tienen entre sus objetivos el reforzamiento de lazos verticales. A partir de 1880 la situación se simplifica, y las asociaciones voluntarias sin objetivos específicos pierden terreno en favor de organizaciones que defienden esferas de intereses particulares. Al mismo tiempo, se plasma una dicotomía local/nacional que atraviesa las formas asociativas, cuestión central en la historia de la sociabilidad ${ }^{13}$.

Quizá uno de los problemas relativos a la transformación del concepto de sociabilidad en Italia es la cronología. Si se analiza la Italia post-unitaria, resulta evidente el retraso tanto en el proceso de industrialización como en la afirmación del capitalismo en el campo. La sociabilidad obrera en ambos espacios fue más el resultado del modelo de la lucha de clases que del modelo integrador ${ }^{14}$. El sistema de "vasos comunicantes» y de la transmisión de la política de arriba hacia abajo, pasando por estructuras intermedias, parece haber cuajado más en las áreas urbanas con un fuerte artesanado y en zonas rurales caracterizadas por el predominio de la pequeña propiedad, como en la Padania. Sin embargo, esto no será lo habitual pues, frente a la Francia del último tercio del siglo XIX, donde el proceso de "ruralización» supone una mayor difusión de la pequeña propiedad, en Italia muchas zonas siguen dominadas por la gran propiedad con un gran número de jomaleros. Así, se puede hablar de una sociabilidad conflictiva, producto de la historia italiana marcada por la tardía unificación, que ha inducido a las diversas clases sociales más a la disociación que a la adhesión orgánica al nuevo Estado. Por eso, según Malatesta, es difícil encontrar en la historia política de la Italia del Ochocientos el carácter profundo del concepto

11. "Sociabilità e associazionismo...", pp. 35-36.

12. Idem, pp. 37-38.

13. Idem, p. 39.

14. Ramelia, F, "Aspetti della sociabilità operaia nell'Italia dell'Ottocento", en MAIULlari, M. T. (a cura di), Storiografia francese e italiana a confronto sul fenomeno associativo durante XVIII e XIX secolo, Torino, Fondazione Luigi Einaudi, 1990, pp. 171-178. 
de sociabilidad que se ha manifestado en Francia a través del lazo con la idea de democracia y de nación ${ }^{15}$.

Durante la década de 1990, la publicación en italiano de algunas de las obras de Agulhon coincide con lo que Ridolfi ha calificado como la reciprocita degli sguardi entre la historiografía italiana y la francesa, si bien puede decirse que, al mismo tiempo, conviven dos metodologías en el país trasalpino: una orientada hacia la historiografía francesa y otra hacia la alemana. Opciones que tienden a legitimar diversas interpretaciones de la «civilización» italiana. La primera pone el acento en las estructuras formales e informales de la sociabilidad, destacando la implicación de sectores de las clases populares, más urbanos que rurales, condicionados por los eventos de 1848. La segunda corriente historiográfica centra su atención en el difícil surgimiento del asociacionismo burgués institucionalizado en el contexto de un Estado centralizado y con recurrentes tentaciones autoritarias $^{16}$. En línea con esta orientación, Quaderni Storici dedica en 1991 un monográfico al asociacionismo de las elites en Italia. Los ensayos aquí reunidos no tratan sobre las formas de la sociabilidad o sobre los modos de construcción de la opinión pública, sino que el motivo conductor es la consideración de que las asociaciones formales aparecidas en la primera mitad del Ochocientos -el casino y después el círculo- reconstruyen los "lenguajes de estratificación» ${ }^{17}$. Estas asociaciones funcionan como instrumentos que subrayan los invisibles límites que cortan en muchas direcciones el espacio social. Lo hacen por medio de las normas estatutarias que regulan el acceso de los socios: el pago de una cuota y la cooptación como mecanismos de admisión significan la capacidad para excluir más que la voluntad para admitir. Pero lo más interesante de estas sociedades es que hasta el año 1880 funcionan como formas de organización pre-partido, al ser ámbitos de formación de las opiniones y definiciones de las orientaciones políticas de los grupos de notables. En ese ámbito, los círculos aparecen en un punto intermedio entre Estado y sociedad, y convirtiéndose en centros operativos de la política local ${ }^{18}$.

Dentro de la misma corriente historiográfica, se publica en 1992 Milano borghese, de Meriggi ${ }^{19}$. La capital lombarda a lo largo del siglo XIX es el espacio emblemático donde se estudian los recorridos y alineamientos de la sociedad

15. MALATESTA, M., «Il concetto di sociabilità nella storia politica italiana dell'Ottocento", Dimensioni e problemi della ricerca storica, $n{ }^{\circ} 1$ (1992), pp. 59-71.

16. Ridolf, M., "Maurice Agulhon en Italia», en Política y sociabilidad. En torno a Maurice Agulhon. Actas del congreso organizado por la Casa de Velázquez de Madrid, el 5 y 6 de febrero de 2001 (en prensa).

17. "Elites ed associazioni nell'Italia dell'Ottocento", Quaderni Storici, n. ${ }^{\circ} 77$ (1991), p. 359.

18. En la revista encontramos, entre otros, artículos sobre: los clubes masculinos de Turín, el Casino Vechio de Trieste, las sociedades comerciales de Florencia, y las asociaciones y comités electorales de Piacenza. Igualmente interesante es RomanelLI, R., "Il casino, l'accademia, il circolo. Forme e tendenze dell'associacionismo d'elite nella Firenze dell'Ottocenton, en MaCRY, P. e MAssafra, A. (a cura di), Fra storia e storiografia. Scritti in onore di Pasquale Villani, Bologna, Il Mulino, 1994, pp. 809-851.

19. Meriggi, M., Milano borghese. Circoli ed elites nell'Ottocento, Venezia, Marsilio, 1992. Del mismo autor: Forme di sociabilità nelle società tradizionali e nella modernità, Viareggio, Baroni, 1997. 
burguesa. Mientras declina la larga fase del predominio aristocrático, todavia vigoroso en los años de la Restauración, adquieren forma los círculos, expresión de los hombres de negocios a los que se sumará el variado mundo de las profesiones liberales. Esta elite constituirá una suerte de "gobierno en la sombra" de la ciudad, para acentuar con el tiempo su propio poder y prestigio, hasta asumir de hecho el gobierno después de la Unificación. A través de la red de sus instituciones privadas, los notables milaneses no sólo codificaron un inédito estilo de vida, imprimiendo entre otras cosas una nueva clave en las relaciones entre hombres y mujeres ${ }^{20}$, sino que mantuvieron el monopolio del juego de asociación, incluso cuando a fines de siglo la pequeña burguesía y los obreros se abrieron a la dinámica asociativa.

Perfiles similares muestra el caso de Nápoles ${ }^{21}$, si bien puede apuntarse una diferencia antes y después de la Unificación. A lo largo de la Restauración, el cuadro que aparece es el de una realidad marcada por la ausencia de iniciativa privada y por la excesiva oferta pública. El Estado organiza la sociabilidad académica, la científico-económica y la recreativa, abundando los casinos, convertidos en lugares de encuentro mundano y de definición institucional de las diferencias de clase. Y aunque desde el años1840 este modelo es puesto en discusión, ante la exigencia de una sociabilidad más diversa que se extiende por toda la península, la ausencia de libertad de imprenta y de asociación, especialmente en el Mezzogiorno, limitan el protagonismo del asociacionismo en la esfera pública. Con la unidad de Italia, y la aplicación del artículo 32 del Statuto albertino que establece una mayor libertad de asociación, el panorama cambia radicalmente ${ }^{22}$. En Nápoles se multiplican los clubes, los círculos y los comités electorales. Su ámbito es local, aunque se inspiran en un modelo general: exclusión de las mujeres, cuota de inscripción como instrumento de selección y pertenencia de sus miembros a la elite. Sin embargo, a partir de 1880 coexisten en la capital de Campania dos tipos de asociaciones elitistas: unas con un fuerte grado de homogeneidad y un acceso muy restringido, y otras más abiertas y menos rígidas. En las primeras se encuentran personas con el mismo status, que a menudo ejercen la misma profesión, con lazos de parentesco y que viven

20. Excluidas inicialmente las mujeres del espacio asociativo, poco a poco se van integrando en sociedades musicales, filantrópicas y otras como el "Touring Club». En ocasiones, crean su propias asociaciones, como el Círculo Filológico, frente al homónimo masculino. MeriGGi, M., "Milano...", pp. 196-216. En el Abruzzo, a comienzos del siglo XX, se corrobora la importante presencia de la mujer en el asociacionismo asistencial, a la par que adquiere un cierto protagonismo político. FolLACCHO, S., "Esempi di notabilato femminile in Abruzzo tra Otto e Novecento", Abruzzo Contemporaneo, n. ${ }^{\circ}$ 10-11 (2000), pp. 149-162. Una reflexión general sobre la Italia liberal en RudolfI, M., "L'apprendistato alla cittadinanza. Donne e sociabilità popolare nell'Italia liberale», Meridiana, n. ${ }^{\circ} 22-23$ (1995), pp. 67-97.

21. Cacliotı, D. L., Associazionismo e sociabilitè d'elite a Napoli nel XIX secolo, Napoli, Liguori, 1996.

22. Un interesante análisis sobre el derecho de asociación y su significado atendiendo a la posición de los juristas y de los políticos italianos del siglo XIX en MERIGGr, M., "Dalla restaurazione all'età liberale. Per una storia del concetto di associazione in Italia", en GHERARDi, R. e GozzI, $G$. (a cura di), I concetti fondamentali delle scienze sociali e dello Stato tra Otto e Novecento, Bologna, Il Mulino, 1992, pp. 87-106. 
en el centro de la ciudad; en las segundas aparece una clase en formación que, lejos de conocerse, experimenta a través de la sociabilidad recreativa y cultural nuevas posibilidades de relación; el reclutamiento de sus socios se hace en un área geográfica más extensa de la urbe, predominando la amplitud del espectro profesional sobre el parentesco.

Dentro de lo que ha sido la orientación hacia la historiografía francesa, cabe destacar uno de los primeros trabajos de Ridolfi sobre el movimiento republicano ${ }^{23}$. En él pone de manifiesto el crecimiento del republicanismo en Italia desde el año 1830, al que reconoce un carácter precursor en la creación de modernas asociaciones políticas. El estudio del republicanismo italiano permite observar, a través de diversos aspectos, la naturaleza de la estrecha pero no lineal correlación existente entre extensión de las formas de sociabilidad y desarrollo del asociacionismo político. El autor centra su investigación sobre la Romaña, por ser la región más politizada y subversiva a nivel nacional, y por haber sido el epicentro del asociacionismo mazziniano y, a partir del año 1880, una de las cunas del partido socialista. Los primeros cambios en la región se operan a partir de 1846, tras la elección del papa Pío IX, quien promueve varias reformas que permiten la aparición de unioni ausiliarie de barberos, zapateros, albañiles, con el objetivo de darse socorro mutuo ${ }^{24}$. Dichas sociedades tienen un particular relieve por el carácter democrático con el que sus estatutos informaban sobre la elección de los dirigentes y la organización de su vida interna, representando una importante innovación en las prácticas asociativas ${ }^{25}$. Pero fue en el bienio 1848-49 cuando en Romaña, como en otros muchos centros del Estado Pontificio, se registró la constitución de círculos populares. Éstos van a representar el primer instrumento público de participación popular en vida política en la Italia del Ochocientos. Ahora, las sedes de los círculos o los teatros eran lugares donde se podía hablar abiertamente, discutir de política y hacer propuestas a través de la democracia del voto. El pueblo se apropiaba de la ciudad y la política scendeva in piazza ${ }^{26}$. Al margen de los espacios más o menos formales de las asociaciones populares, Ridolfi subraya la importancia que adquieren, con la unidad, la celebración de los aniversarios, de forma especial la República romana y la onomástica de Garibaldi y de Mazzini. Con esta última, los republicanos pretendían apropiarse de la festividad católica a través de la definición

23. Ridolfi, M., «Sociabilità e politica nell'Italia dell'800. Aspetti dello sviluppo associativo del movimento repubblicano fra restaurazione e primi anni post-unitari», en MAIULLARI, M. T. (a cura di), Storiografia francese e italiana a confronto sul fenomeno associativo durante XVIII e XIX secolo, Torino, Fondazione Luigi Einaudi, 1990, pp. 179-209.

24. Sobre las sociedades de socorro mutuo puede verse: SolDanl, S., "La mappa delle società di mutuo soccorso in Toscana fra l'Unità e la fine del secolo", en BuGARAN, A. (a cura di), Istituzioni e borghesie locali nell'Italia liberale, Milano, Franco Angeli, 1986, pp. 247-294. CamURr, R. (a cura di), Censimento storico delle società di mutuo soccorso del Veneto, Venezia, Regione Veneto, Venezia, 2003, 2 vols.

25. RrdoLFr, M., «Sociabilità e politica...», p. 185.

26. Idem, pp. 189-190. 
de nuevos símbolos y significados ${ }^{27}$. Por otra parte, el movimiento republicano buscó ampliar el consenso popular y la difusión de sus ideas a través de grupos de música y de canto, destacando además, por su significado, el interés demostrado hacia el asociacionismo deportivo, alimentado por el espíritu gimnásticomilitar del garibaldinismo.

Si todo lo anterior tenía lugar en ámbitos urbanos, en los centros rurales de la Romaña surgen las llamadas Cameracce. Aparecen antes de 1860 con la proliferación de las asociaciones de reventa del vino para evitar los onerosos impuestos aplicados al consumo del alcohol. Alquilado un local, los campesinos y los artesanos se aseguraban un lugar de encuentro y de ocio, que poco a poco se convirtieron también en un lugar de reunión política. En este sentido, la ley electoral de 1882 propició la multiplicación, no sólo de comités electorales, sino también de mítines y banquetes que van a marcar una etapa importante en la transformación de las reuniones de masas no religiosas, ampliando los lugares ocupados por la política. El mitin adquiere, así, el carácter de «escuela de las masas ${ }^{28}$, algo de lo que serán conscientes desde el primer momento, a diferencia de los republicanos, las asociaciones socialistas, al comprender que aquéllos ayudan a conformar un partido de masas y que tienen un objetivo eminentemente político ${ }^{29}$.

Relevancia singular va a tener igualmente la masonería en los procesos de politización. Mediante su investigación sobre Toscana, Conti interpreta que, en la confluencia de muchas organizaciones laicas y democráticas, la masonería se convierte, durante la segunda mitad del siglo XIX, en un punto de multiplicación de las formas de sociabilidad democrática y es el verdadero tejido conductor de las mismas ${ }^{30}$. El florecimiento en los años finiseculares de asociaciones parapolíticas, por lo demás de fe republicana, que aceptaron los rituales y las formas organizativas masónicas, puede ser leída también como una especie de medida cautelar adoptada para intentar sustraerse de la represión del Estado. Al mismo tiempo, amplios sectores del asociacionismo masónico fueron determinantes en la obra de legitimación del nuevo Estado y de «educación a la política». Un ejemplo de ello lo tenemos en las circulares del primer período postunitario, en las que se invitaba a los miembros de las logias, fuesen cuales fuesen sus ideas políticas, a participar en las elecciones ${ }^{31}$.

27. Idem, p. 197.

28. Idem, p. 207.

29. Rrdolfi, M., Il PSI e la nascita del Partito di Massa, 1892-1921, Roma-Bari, Laterza, 1992. Especialmente el capítulo $\mathrm{V}$.

30. ConTI, F, Laicismo e democrazia. La massoneria in Toscana dopo l'Unità (1860-1900), Firenze, Centro editoriale toscano, 1990. Con carácter comparativo, véase "Massoneria e politica in Europa fra Ottocento e Novecento (Italia, Spagna e Portogallo)", monográfico de Memoria e Ricerca, nueva serie, n. ${ }^{\circ} 4(1999)$.

31. ConTI, F, L'Italia dei democratici. Sinistra risorgimentale, massoneria e associazionismo fra Otto e Novecento, Milano, Franco Angeli, 2000, pp. 119 y 196. Véase también, ConTr, F., "Fra patriottismo democratico e nazionalismo. La massoneria e l'idea di nazione nell'Italia liberale», Contemporanea, II, n. 2 (1999), pp. 221-248. 
Conti destaca, por otra parte, el interés que tienen las sociedades de veteranos de guerra que, entre la unidad y la Primera Guerra Mundial, representan un elemento de gran relieve en el tejido asociativo de Toscana. Se trata de lo que el autor considera un "asociacionismo de frontera», difícil de situar entre los cánones tradicionales de la historia del mutualismo, en los que no se agota su finalidad, y que ha recibido escasa atención en las obras dedicadas a la evolución de la cultura laica en la Italia liberal. Sin embargo, es un ámbito de la investigación, sobre todo en lo referido a su universo ideológico y ritual, que ha comenzado a ganar terreno en el tema de la afirmación del concepto de patria durante la segunda parte del Ochocientos. Cabe decir que las asociaciones de veteranos se convirtieron en el primer fenómeno organizativo popular a nivel nacional. Con sus uniformes, sus banderas, sus desfiles, con la rigurosa ritualidad de las ceremonias fúnebres, estas sociedades contribuyeron a dotar los ideales patrióticos de precisos referentes simbólicos, e hicieron también que se difundiesen y radicasen en el imaginario colectivo. De todos modos, todavía no está claro si esta sociabilidad fue integradora, opositora al régimen, o si supuso una vía intermedia y ambivalente ${ }^{32}$.

Tras la unidad, y con el paso del tiempo, se va a desarrollar en Italia, como en otros países europeos, una dinámica de laicización donde pierde peso el espacio privado en beneficio del público y adquiere significado la sociabilidad no formalizada. Sin embargo, hasta mediados de los años 1990, apenas se ha aplicado en Italia el concepto de sociabilidad para tratar la problemática de la secularización. Y los estudios de la sociabilidad tienden a mirar los procesos de secularización a la luz de los datos obtenidos en investigaciones tradicionales, verificando superficialmente la difusión de las ideas laicas en los circuitos asociativos o la diferencia entre asociaciones laicas y confesionales ${ }^{33}$.

Mayor éxito han tenido los análisis sobre la ritualidad civil, en donde se destaca la importancia de las fiestas patrióticas en la población para reafirmar su pertenencia a la nación. En 1995, Memoria e Ricerca publica un dossier sobre «las transformaciones de la fiesta» en Francia, Italia y España. Los autores consideran que, si bien cada fiesta la inventa y la administra un grupo, cuando ésta se desarrolla en lugares abiertos, como las plazas, conforma un sistema de comportamiento del que se apropian las comunidades, introduciendo identidades propias y rediseñando espacios y tiempos de su historia, real o imaginaria. En el siglo XIX, en particular, la fiesta se convierte en el terreno privilegiado de confrontaciones y antagonismos entre las nuevas formas asociativas del mundo burgués y popular que van difundiéndose en la sociedad europea ${ }^{34}$. EI significado de la fiesta registra una cesura bastante neta entre la representación tradicional de la comunidad, esencialmente religiosa y folclórica, y las transfor-

32. Contr, F., L'Italia dei democratici..., pp. 193-229.

33. FINCARDI, M., "Sociabilità e secolarizzazione negli studi francesi e italiani», Italia Contemporanea, n. ${ }^{\circ} 192(1993)$, pp. 511-528.

34. "Le trasformazioni della festa. Secolarizzazione, politicizzazione e sociabilità nel XIX secolo (Francia, Italia, Spagna)", monográfico de Memoria e Ricerca, n. ${ }^{\circ}$ (1995), p. 7. 
maciones de rituales públicos en sentido laico y civil. En Italia, con el bienio revolucionario de 1848-1849 y después con el de la unificación de 1859-1861, las prácticas de la sociabilidad cotidiana registran un inédito impacto en la esfera pública de la política ${ }^{35}$. Antes que las reformas electorales y la organización de los intereses sociales transformen el cuadro de referencia y encuentren respuesta en la formación de las modernas estructuras de partido, el acceso a la política se produce en virtud de la implicación colectiva en los primeros ritos festivos y civiles públicos. Conmemoraciones y funerales son promovidos y guiados por las redes de asociaciones, más que a través de los mecanismos elitistas de la representación ${ }^{36}$. Durante el Risorgimento cobrará especial importancia el teatro como espacio de la sociabilidad informal. En 1848 deja de ser el lugar de orden concebido por las autoridades de la Restauración, y pasa a convertirse en un espacio de subversión, recuperando formas comunicativas y litúrgicas, de forma especial con las representaciones operísticas ${ }^{37}$.

En la historiografía sobre el imaginario político de los italianos, sin embargo, todavía es insuficiente, como señala Ridolfi, el espacio reservado a los símbolos y a las imágenes a través de las cuales la tradición democrático-republicana fue asumiendo una forma estética propia en los tiempos de la monarquía ${ }^{38}$. A diferencia de Francia, en Italia fue más conflictiva y más tardía la relevancia de la simbología y de la estética republicanas en pugna por afirmar una cultura cívica. Dos serán los elementos fundamentales: la memoria de Mazzini, a través de la representación en ritos y monumentos públicos, y la transformación de la República Romana de 1849, de evento revolucionario, en el principal símbolo de la tradición republicana. La rivalidad entre demócratas y monárquicos por el control de las memorias del Risorgimento y de su representación pública es evidente desde los primeros años post-unitarios. Él caso más significativo es el culto a Garibaldi que, si bien aparece con una connotación anticlerical, al unirse a la memoria de Vittorio Emmanuelle, adquiere una función de defensa del régimen monárquico. En cambio, la figura de Mazzini fue marginada por el Gobierno respecto a la pedagogía patriótica. Y no es casual que en Roma, lugar de gran impacto simbólico, el monumento a la memoria del político demócrata, si bien fue autorizado por ley en 1890, no se construyera hasta $1949^{39}$.

35. Porciani, I., La festa della nazione. Rappresentazione dello Stato e spazi sociali nell'Italia unita, Bologna, Il Mulino, 1997.

36. RIDOL.H, M., "Lugares y formas de la vida cotidiana en la historiografia italiana", en CASTELLS, I. (ed.), "La Historia de la vida cotidiana», Ayer, n. ${ }^{\circ} 15$ (1995), pp. 71-100.

37. BANTI, A. y BIzzocchI, R. (a cura di), Immagini della nazione nell'tialia del Risorgimento, Roma, Carocci, 2002, pp. 133-156. Véase también BAIONI, M., "Identità nazionale e miti del Risorgimento nell'Italia liberale. Problemi e direzioni di ricerca", Storia e problemi contemporanei, XI, n. ${ }^{\circ} 22$ (1998), pp. 17-40.

38. Ridolfi, M., "El culto de la República en los tiempos del rey. Lugares de la memoria y símbolos republicanos en la Italia liberal», Historia Social, n. ${ }^{\circ} 29$ (1997), pp. 111-128.

39. Será Roma, lógicamente, el lugar privilegiado donde el poder político liberal identificará los principios de su legitimación, con la construcción de un nuevo espacio urbano poblado de monumentos y animado por itinerarios patrióticos. Esto tendrá un doble significado, porque Roma no sólo era la capital del Estado, sino también el lugar donde permanecía irreductiblemente hos- 
Dentro de la temática de "las religiones de la patria», y con una amplia perspectiva, Ridolfi hace un recorrido, en un reciente libro, por «los días de Italia», analizando la identidad italiana a través de las fiestas nacionales ${ }^{40}$. Éstas representan un observatorio ideal para ver cómo interaccionan en el discurso político los diversos símbolos del Estado - la fundación, la memoria del pasado, la forma de gobierno, la Constitución-y de la sociedad -la patria, la imagen del país-. Todo ello nos traslada a los aspectos emocionales de la política a través de la correlación entre religiones tradicionales y religiones seculares. En Italia, no obstante, durante la época contemporánea, la formación de una religión civil ha pagado los efectos contradictorios, no sólo por la relación entre las aspiraciones de progreso y el retraso social, sino sobre todo por la competencia entre las instituciones y la Iglesia. Así, la relación entre historia y memoria en la construcción de la identidad nacional es indagada en relación con el complejo sistema de símbolos patrióticos y rituales civiles que han caracterizado las tres Italias -liberal, fascista, republicana-. A esto sigue la atención hacia las fiestas de la nación que hoy día representan los principales rituales civiles del Estado: el 4 de noviembre -final de la "Gran Guerra»-, el 25 de abril -día de la Liberación-y el 2 de junio -nacimiento de la República-.

Una vez recorrido el panorama historiográfico italiano sobre la sociabilidad, puede afirmarse que destaca, hoy día, por la diversidad de temas y por el rigor en su tratamiento. Como es lógico, y los historiadores italianos lo ponen de manifiesto, hay todavía campos que merecen investigaciones en profundidad. El mundo campesino es uno de ellos ${ }^{41}$, y el objetivo sería, desde un enfoque interdisciplinar, poner el espacio rural en el análisis de los procesos de politización y de integración del Estado nacional. En este sentido, y por último, conviene no perder de vista la existencia de las "diversas Italias", y la necesidad de explicar los rasgos que las delimitan.

til la presencia del papado. Véase ToBIA, B., Una patria per gli italiani, Roma-Bari, Laterza, 1991. También, NAsto, L., Le feste civili a Roma nell'Ottocento, Roma, Grupo Editoriale Internazionale, 1996. Sobre los lugares de la memoria son fundamentales: IsNENGHI, M. L'Italia in piazza. I luoghi della vita pubblica dal 1848 ai giomi nostri, Milano, Mondadori, 1994; ISNENGHI, M. (a cura di), I luoghi della memoria. Strutture ed eventi dell'Italia unita, Roma-Bari, Laterza, 1997.

40. Ridolfr, M., Le feste nazionali, Bologna, Il Mulino, 2003.

41. Dos referencias destacables son: FinCARDI, M., "Culture comunitarie e moderni conflitti sociali nell'Italia rurale di fine XIX secolon, en La politisation des campagnes au XIX siede. France, Italie, Espagne, Portugal et Grece, Roma, École Française de Rome, 2000, pp. 221-257. PÉcour, G., "Feste unitarie e integrazione nazionale nelle campagne toscane (1859-1864)", Memoria e Ricerca, n. ${ }^{\circ} 5$ (1995), pp. 66-81. 